Preprint typeset in JINST style - HYPER VERSION

\title{
MegaPipe astrometry for the New Horizons spacecraft
}

\author{
Stephen D. J. Gwyn ${ }^{a *}$ \\ ${ }^{a}$ Canadian Astronomy Data Centre, \\ 5071 West Saanich Rd, Victoria BC, V9E 2E7, Canada \\ E-mail: stephen.gwynenrc.ca
}

\begin{abstract}
The New Horizons spacecraft, launched by NASA in 2006, will arrive in the PlutoCharon system on July 14, 2015. There, it will spend a few hours imaging Pluto and its moons. It will then have a small amount of reserve propellant which will be used to direct the probe on to a second, yet to be discovered object in the Kuiper Belt. Data from the MegaPrime camera on CFHT was used to build a precise, high density astrometric reference frame for both the final approach into the Pluto system and the search for the secondary target. Pluto currently lies in the galactic plane. This is a hindrance in that there are potential problems with confusion. However, it also a benefit, since it allows the use of the UCAC4 astrometric reference catalog, which is normally too sparse for use with MegaCam images. The astrometric accuracy of the final catalogs, as measured by the residuals, is 0.02 arcseconds.
\end{abstract}

KEYWORDS: Detectors for UV, visible and IR photons, Image processing, Data reduction methods.

\footnotetext{
${ }^{*}$ Corresponding author.
} 


\section{Contents}

1. Introduction 1

2. Method 1

3. Tests 4

\section{Introduction}

MegaCam [1] is a mosaic camera with a one degree field of view on the Canada France Hawaii Telescope (CFHT). It consists of 36 E2V CCD detectors. MegaPipe [2] is the MegaCam data processing pipeline at the Canadian Astronomy Data Center. MegaPipe began processing all publicly available MegaCam data in 2005. Since then, it has produced over 3000 square degrees of stacked images and catalogs. It also processes PI data on request and handles data from the CFHT Large Programs.

The New Horizons [3] spacecraft was launched by NASA in 2006 and is currently en route to Pluto. In 2015, on July 14, it will fly through the Pluto-Charon system for a few hours. After the Pluto flyby, the space craft will have a small amount of reserve propellant. This will be used to alter course toward a second Kuiper Belt Object (KBO). The ongoing search [ $₫$ ] for this object involves data from the Subaru and Magellan as well as CFHT. The MegaPipe images are being used as the astrometric reference frame for the search. Between 45 and 6 days before the encounter, a search will be conducted of the Pluto-Charon system for potentially hazardous debris using LORRI, the onboard detector. Again, MegaPipe data is being used as the astrometric reference for this search.

This paper describes the production of the astrometric reference catalogs used for both the search for the second KBO target and the debris search. The construction of these catalogs was complicated by the fact that Pluto is currently crossing the galactic plane. The stellar density in this region of the sky is extremely high for the most part, but drops off sharply in places due to the presence of dust clouds in the field, as shown in figure 11. There is a strong potential for confusion when matching stars in the images to stars in the astrometric reference catalog.

\section{Method}

The MegaPipe astrometric calibration method has been described elsewhere (e.g., [2]], [阿). Consequently, the following discussion will be brief, except to emphasize the inherent strengths of the standard MegaPipe procedures when dealing with crowded images and what recent improvements have been incorporated to those procedures to deal with the crowded New Horizons field.

The input images from MegaCam have been detrended with Elixir [6] and contain an initial WCS (World Coordinate System) which is used as a starting point. An obverved source catalog is 


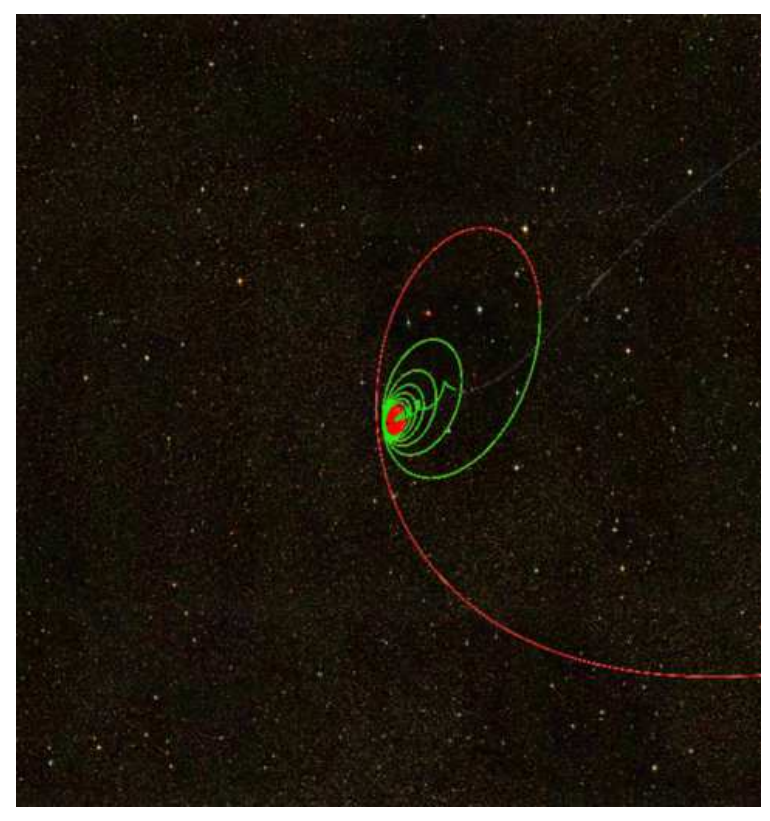

Figure 1. This figure illustrates the approach of New Horizons into the Pluto system. The line shows the position of Pluto, as seen by the space craft, with respect to the background stars. The colors indicate times: green indicates 45 and 6 days pre-encounter when the search for hazardous debris will occur. The image is one degree on a side.

generated for each image with SExtractor[7]. MegaCam images are typically much deeper than the external astrometric references catalogs; a magnitude cut is applied to the observed source catalog to minimize the number of potentially confusing sources.

The observed source catalog is matched to the external astrometric reference catalog under the assumption the Elixir linear WCS is close to correct in terms orientation and scaling. Small shifts in RA and Dec are applied to the WCS until the number of matches between the observed source catalog and the external astrometric reference catalog is maximized. When the geometry of the detectors is well known, this simple approach is sufficient, and is more robust in crowded fields than the more sophisticated Fourier transform methods or triangle- and quad-matching methods.

The number of catalog matches is rough indicator of the quality of the WCS match. Normally, off the galactic plane, for a single MegaCam CCD, 30 matches between the external astrometric reference catalog and the observed catalog indicates a successful identification. In the crowded New Horizons fields, the minimum match number was set to 300 matches per CCD. CCDs with fewer matches were flagged for visual inspection. Most such cases the CCD was found to lie on a patch of higher obscuration (i.e. fewer stars). The few remaining cases showed spurious matches and had to be rematched.

Normally, MegaPipe uses the 2MASS [8] catalog as the external astrometric reference, because its high source density. However, since the New Horizons fields are near the galactic center, a shallower but higher precision external astrometric reference catalog can used, namely UCAC4 [9]. When the 2MASS and UCAC4 catalogs are compared over the whole sky, small systematic shifts are visible, as shown in figure 2 . The pattern of the offsets have a 6 degree periodicity in Declination, matching the 2MASS observation strategy, indicating that there may be errors in the 


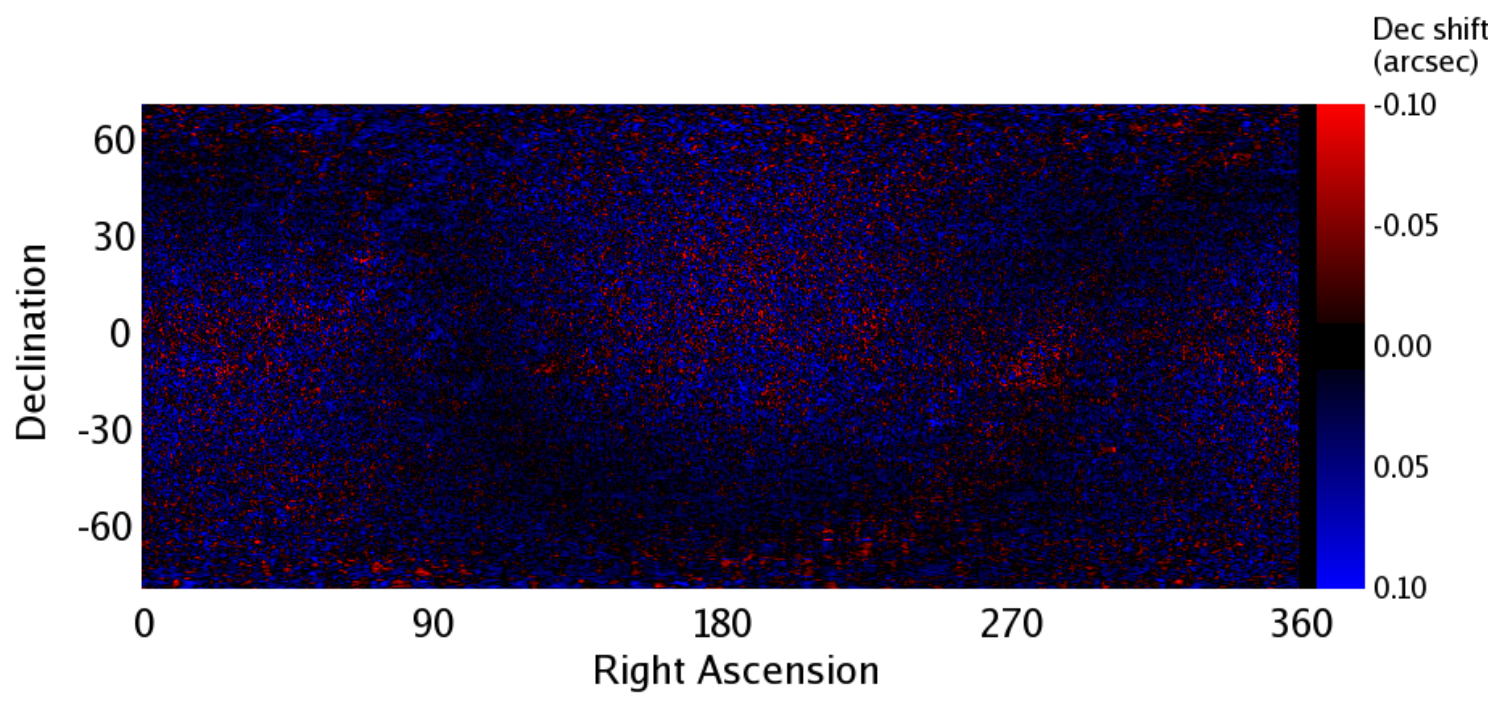

Figure 2. The astrometric residuals between $2 \mathrm{MASS}$ and UCAC4 in Declination. The color coding indicates the size of the astrometric residuals. The galactic plane is visible as a darker swath inidicating smaller astrometric residuals. More subtly, there is a pattern with a 6 degree period in declination, indicating problems with the 2MASS catalog.

2MASS catalog. Similar, but smaller offsets are visible when the SDSS DR9 [10] and UCAC4 catalogs are compared, with the pattern matching the SDSS swathes.

Once the observed source catalog and the external astrometric reference catalog are matched, the field distortion can be measured. MegaPipe models the focal plane distortion as

$$
R=r\left(1+a_{2} r^{2}+a_{4} r^{4}\right)
$$

where $r$ is the measured radius from the center and $R$ is the true radius. This means that only two coefficients ( $a_{2}$ and $\left.a_{4}\right)$, which can be determined once for the whole mosaic, are needed to describe the distortion. In typical astrometric packages, a third-order polynomial in $x$ and $y$ pixel position is computed, requiring 20 parameters for each detector of a mosaic. In the case of MegaCam, this would mean 720 coefficients. MegaPipe instead only models the linear part of the WCS at the chip level. This means that there only 6 parameters per chip, plus 2 for the higher order distortion, for a total of 218. Keeping the number of parameters relatively small prevents spurious matches in crowded fields, and allows more robust fitting when there are steep gradients in the source density of the external astrometric reference catalog. The values of $a_{2}$ and $a_{4}$ are calculated for each MegaCam exposure, although the values change only very slightly from between exposures. The values of $a_{2}$ and $a_{4}$ are initially set to nominal values and the linear terms for each chip are computed by linear regression. The astrometric residuals are computed using the current fit. The values of $a_{2}$ and $a_{4}$ are then allowed to vary until the astrometric residuals are minimized.

While the distortion is expressed internally in MegaPipe as shown in equation 2.1, it is expressed as a polynomial using the PV_nn WCS keywords in the FITS headers. This conversion merely re-expresses the distortion; no additional fitting is actually done, despite the apparent increase in the number of coefficients. 


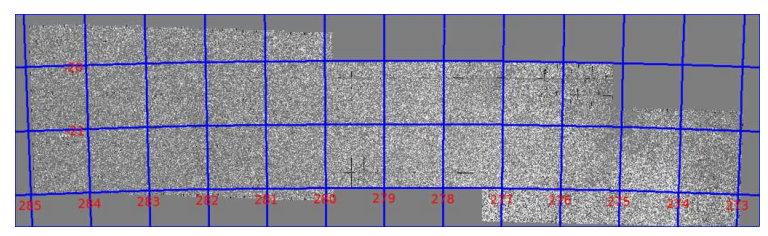

Figure 3. This image shows the 20 square degrees that are being searched for a secondary KBO target for the New Horizons probe.

This initial matching and fitting procedure is applied to the input images. After the WCS has been computed, it is applied to the observed source catalogs to convert the $x, y$ pixel coordinates to RA and Dec. The RA/Dec catalogs are then combined to produce an intermediate astrometric catalog covering the whole field. Sources common to more than one catalog are identified and their coordinates from different images averaged to increase the positional accuracy.

The matching and fitting procedure is repeated on the input images, but this time, instead of using UCAC4 as the external astrometric reference, the intermediate catalog is used. The images, with updated WCS in their headers are combined using SWarp ${ }^{1}$. SExtractor is run on the combined stack to generate the final astrometric catalog. The SExtractor parameters are changed slightly relative to the default MegaPipe configuration to deal with the crowding. In particular, the minimum contrast parameter for deblending, DEBLEND_MINCONT, is set to a much lower value. This image combination step effectively merges the astrometric measurements from each image at the pixel level. This final astrometric catalog can be used to calibrate the individual input images a final time so that they can be used for KBO searches.

This method was applied to the KBO search fields. The resulting image is shown in figure 3 .

The Pluto hazard detection field has data taken in multiple bands. The images taken in the $\mathrm{r}$-band were processed first. The $g$ - and $i$ - band data were calibrated using the r-band image as the astrometric reference. Despite the difference in wavelength between the three bands, chromatic differential refraction will not introduce any systematic effects in the astrometry of the $g$ and $i$ images relative to the $r$-band image as a function of position. The positions of stars will shift in azimuthal direction function of their color, but because the images were taken a low airmass, the amount is small, $\sim 0.004^{\prime \prime}$ per magnitude in $(g-i)$. The gri images were combined to produce the color image indicating the background in figure 1.

\section{Tests}

The catalogs from the calibrated individual images were matched against each other and against the final catalogs and the residuals examined. For the Pluto encounter field, the catalogs in the gri bands were also checked against each other. The astrometric residuals were typically $0.02^{\prime \prime}$, as shown in figure 4 . The unmodified MegaPipe pipeline produces astrometric residuals that are typically $0.04^{\prime \prime}$. Systematic errors would be readily detectable in this figure, particularly in the whisker plot in the upper left panel, where they would show up as groups of parallel lines.

The positions and magnitudes of the Pluto encounter field catalog were used to simulate an image taken with LORRI. The catalog positions were convolved with LORRI's PSF. The simu-

\footnotetext{
${ }^{1}$ http://www.astromatic.net/software/swarp
} 

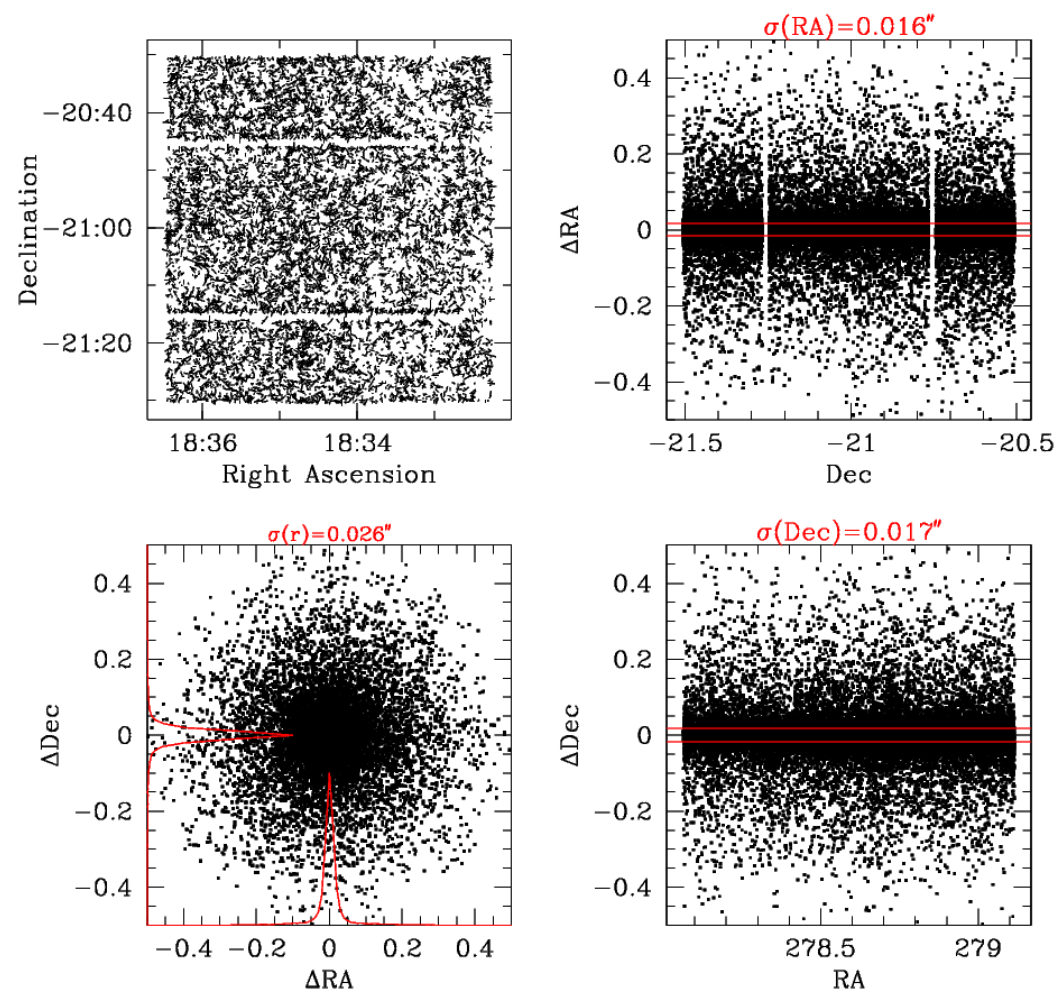

Figure 4. The top left panel shows the astrometric residuals as a whisker plot. The lines show the orientation and relative amplitude of the residuals. The amplitude of the residuals is greatly exaggerated. The bottom left panel shows the residuals in RA and Dec as a scatter plot. The panels on the right show the residuals in RA and Dec as function of Dec and RA respectively. While the random errors are on the order of $0.02^{\prime \prime}$, there is no evidence for any systematic errors.

lated image was subtracted from a real image, after making small adjustments for the spacecraft's pointing and roll angle. Although small residuals (both positive and negative) reveal a bandpass mismatch between MegaCam and LORRI, the alignment is perfect, as shown in figure 5 .

\section{References}

[1] Boulade, O. et al. MegaCam: the new Canada-France-Hawaii Telescope wide-field imaging camera Proceedings of the SPIE 4841 (2003) 72.

[2] Gwyn, Stephen D. J., MegaPipe: The MegaCam Image Stacking Pipeline at the Canadian Astronomical Data Centre, PASP 120 (2008) 212.

[3] Stern, S. A. The New Horizons Pluto Kuiper Belt Mission: An Overview with Historical Context Space Science Reviews 140 (2008) 3 [astro-ph/07094417]

[4] Kavelaars, J. J. et al. Searching for Kuiper Belt Object Flyby Targets for the New Horizons Spacecraft AAS/Division for Planetary Sciences Meeting Abstracts (2012)

[5] Fraser, W. et al. Kuiper Belt Occultation Predictions PASP 125 (2013) 1000.

[6] Magnier, E. A. and Cuillandre, J.-C., The Elixir System: Data Characterization and Calibration at the Canada-France-Hawaii Telescope PASP 116 (2004) 449. 


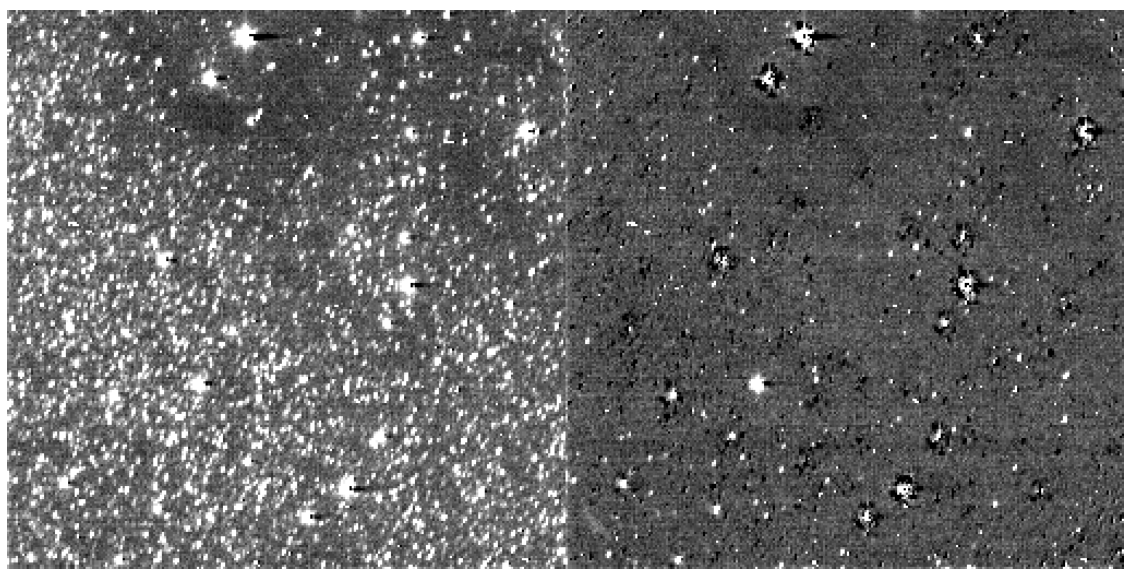

Figure 5. The left panel shows an image taken with LORRI of the Pluto approach field. The right panel shows the residual between this image and a simulated image constructed from by placing predicted LORRI PSFs at the positions and flux levels of objects in the MegaCam astrometric (and photometric) catalog. Most of the stars completely disappear, indicating good astrometry. The remaining residuals are centered on the stars, and indicate that there is a mismatch between the MegaCam and LORRI passbands.

[7] Bertin, E. and Arnouts, S. SExtractor: Software for source extraction Astron. \& Astroph. Suppl. 117 (1996) 393.

[8] Skrutskie, M. F. et al. The Two Micron All Sky Survey (2MASS) Astron. J. 131 (2006) 1163.

[9] Zacharias, N., Finch, C. T., Girard, T. M., Henden, A., Bartlett, J. L., Monet, D. G., Zacharias, M. I. The Fourth US Naval Observatory CCD Astrograph Catalog (UCAC4) Astron. J. 145 (2013) 44.

[10] Ahn, et al. The Ninth Data Release of the Sloan Digital Sky Survey: First Spectroscopic Data from the SDSS-III Baryon Oscillation Spectroscopic Survey Astroph. J. Sup. 203 (2012) 21. 